\title{
The regenerated noise of HVAC elements and its measurement in pipeline track
}

\author{
Pavel Drabek ${ }^{1, *}$ \\ ${ }^{1}$ Tomas Bata University in Zlin, Faculty of Applied Informatics, Department of Automation and Control Engineering, Nad Stranemi \\ 4511, 76005 Zlin, Czech Republic
}

\begin{abstract}
HVAC systems may spread unwanted noise that needs to be reduced. One of the components of wide range noise can be a regenerated noise which is a secondary sound source usually caused by air flowing through a device. This regenerated noise associated with airflow is indeed a dynamic function of velocity. Therefore, HVAC designers try to prevent these problems by sizing ductwork or duct configurations so that air velocity is low. Nevertheless, in certain situations, this approach is not possible and it is necessary to deal with the regenerated noise further. This article discusses an alternative way of measuring regenerated noise of various duct elements in the pipeline track without being connected to a reverberation chamber. It should be emphasized that this method is not new but information about it is very limited and therefore almost unused.
\end{abstract}

\section{Introduction}

Adequate fresh air supply for occupants is an important aspect of building design and construction. Mechanical systems of ventilation are preferred due to their reliability and adjustability as a concern as proper and energy optimal fresh air delivery. [1]. Mechanical ventilating systems is connected both with the air born noise originated in the system itself and/or transfer the noise from the adjacent surrounding areas to the ventilated premises.

Sound and vibration are one of the main observed parameters of HVAC systems [2]. The sound control is then the absolutely essential task to be solved by the HVAC designer and that's why the proper silencer selection is a key part of the HVAC design [3]. This article deals with acoustical properties of some HVAC elements and respective measurement.

The parameters used to describe the acoustical performance of silencers or other devices in HVAC systems are insertion loss, pressure loss and regenerated noise. The pressure loss measurements, which serve for the determination of the total pressure loss coefficient as well as the measurements for the determination of the insertion loss shall be done according to the [4], whilst the acoustic measurements for the determination of the regenerated noise will be carried out in compliance with the [5]. And here comes the problem. The insertion and pressure loss measurement process itself is relatively extensively described, whereas the regenerated noise measurement is a little bit unclear. Although the standards [4][6] mention that it is possible to carry out measurements in the pipeline track, it is further focused only on test stand consist of the duct which is connected to the reverberation chamber. How to properly measure in the pipeline track and then evaluate the regenerated noise itself is no longer mentioned.

Based on the lack of instruction about measurement in the pipeline track in the relevant standards, the intention of this paper is to provide information on measurement and its evaluation of regenerated noise of various duct elements in the pipeline track without being connected to a reverberation chamber.

\section{Regenerated noise and its measurement}

A key approach to noise control is to divide of the entire problem into its basic parts, it means into a source creating the noise, a path transmitting the noise, and a receiver hearing the noise [7]. All these three individual parts are to be observed by HVAC designer or acoustical engineer in the HVAC system design. However, this article deals only with the sound sources in HVAC systems represented by passive elements such as silencers, throttles (dampers), distribution elements, etc.

These passive elements are not by themselves as sources of noise, but when they are exposed to airflow they can become the source of the noise. It is a paradox, for example, in the case of silencers, whose role is to reduce sound transmission from one section of HVAC system to another, it can generate some noise by themselves. The sound power level of the sound source indicates the parameter referred to as the regenerated noise (flow noise or self-generated noise), which is usually given for various face velocities and directions (forward or reverse ones). Since these values represent the amount of sound produced by the duct element, a

\footnotetext{
Corresponding author: pdrabek@utb.cz
} 
lower value will indicate better performance. In other words, regenerated noise level values determine the lowest sound pressure level that can be reached behind a given duct element for a given face velocity. According to [8], regenerated noise increases with increasing pressure drop of the passive elements. Data for regenerated noise levels are provided for each of the third-octave band mid frequencies ranging from $50 \mathrm{~Hz}$ to $10000 \mathrm{~Hz}$.

These values are very important, for HVAC designer, however, manufacturers very often do not publish them, or even do not know them. There exist equations and methods described in the relevant literature to estimate regenerated noise in branches, elbows, splitters, etc. nevertheless, they might be of application sensitive. These theoretical calculations consider, in particular, dimensional parameters, face flow velocity and a certain constant called B. However, the value of this constant B depends primarily on the pressure loss coefficient $\xi$ and some publications state that it is not yet sufficiently established and the value given in [9], as $\mathrm{B}=58 \mathrm{~dB}$ is mainly used. Consequently, this approach may cause very noticeable deviations.

Obviously, the most appropriate way how to find out the regenerated noise values is by measuring. In practice, the regenerated noise of silencers and other duct elements are laboratory tested by substituting the test specimen for an unlined sheet metal duct having the same face dimension. Three basic configurations of the measuring sets are used, the measurement in a reverberation chamber, in the duct behind the silencer and in the surrounding free sound field. The measurements in a reverberation chamber according to [5] are the preferred method to determine the sound power level. However, this type of arrangement is very demanding for space and laboratory equipment. The reverberation room itself must have a relatively large volume to allow the measurement at low frequencies. Moreover, the all surroundings must be made from an acoustically hard material such as smooth concrete. Besides, another space is required for the pipeline track that must be connected to this chamber. Other configurations of measuring sets are no longer so demanding for laboratory equipment as the previous is.

The general philosophy of determining the regenerated noise is as follows. Always two series must be carried out. The first test series for the determination of the ambient noise level with the substitution duct replacing the measuring object. In the second test series, the sound pressure level of the measured objects regenerated noise is determined. During the execution of both series, no other test condition is changed. Afterwards, the sound power level of the regenerated noise $L_{W}$ coming from the measured object is calculated with equation (1) in third-octave bands.

$$
L_{W}=\overline{L_{P}}+D_{t d}+K
$$

Where
$\overline{L_{P}}$ is the spatial energy average of the sound pressure without correction to background noise;

$D_{t d}$ is transmission damping factor;

$K$ is the correction taking into account the calculation of the sound power in the environment where the sound pressure was measured.

The above-mentioned philosophy is, first of all, to organize a test track so as to ensure a quiet flow, then measure the sound pressure in place behind the tested object and then identify places of acoustic discontinuity. These are primarily places with a sudden change in cross-section area where part of the acoustic energy passes through the interface, and the other part will be reflected back [10]. Therefore, it is necessary to consider what amount of acoustic energy has been reflected back to the source. Next, the evaluation procedure differs from what configuration of measuring set was used for the measurement. A description of the performed measurements and a way how to evaluate the data measured in the pipeline track can be found in the following.

\section{Measurement in a pipeline track}

All the general information mentioned in the previous chapter is also valid for pipeline track without its connection to a reverberation chamber. However, it is necessary to look at the measured data from the opposite view. In the reverberation chamber, the measured values of the sound pressure are undervalued due to the reflection of the acoustic energy at the open end of the transmission duct, whereas, in pipeline track, the reflected wave returns back. This means that the $D_{\mathrm{td}}$ parameter in equation (1) cannot be added to the values but subtracted. However, assuming that a special duct element which eliminating the reflection of sound energy, is located in front of the place of acoustic discontinuity, this effect can be almost neglected. These duct elements are called anechoic and the method of their design and assessment is given in [11]. Additional requirements relating to the actual transmission duct. It must be straight and smooth and also sufficiently rigid to prevent breakout noise through its walls [4]. Many things will contribute to the amount of sound that is able to break out of the transmission duct casing, including the shape and size of duct, and the gauge of material used to construct the casing [12]. The most recommended shape is circular or square.

Two types of pipeline track configuration can be used. The first one is track without open ends, schematically depicted in Fig. 1. It is a duct circuit where the air is constantly circulating. The advantage of this closed system is mainly due to its higher resistance to parasitic noise from the environment. On the other hand, the flow regulation of this such a closed system could be a disadvantage. When changing the air flow, it is better to let the fan run at a constant speed and regulate the 


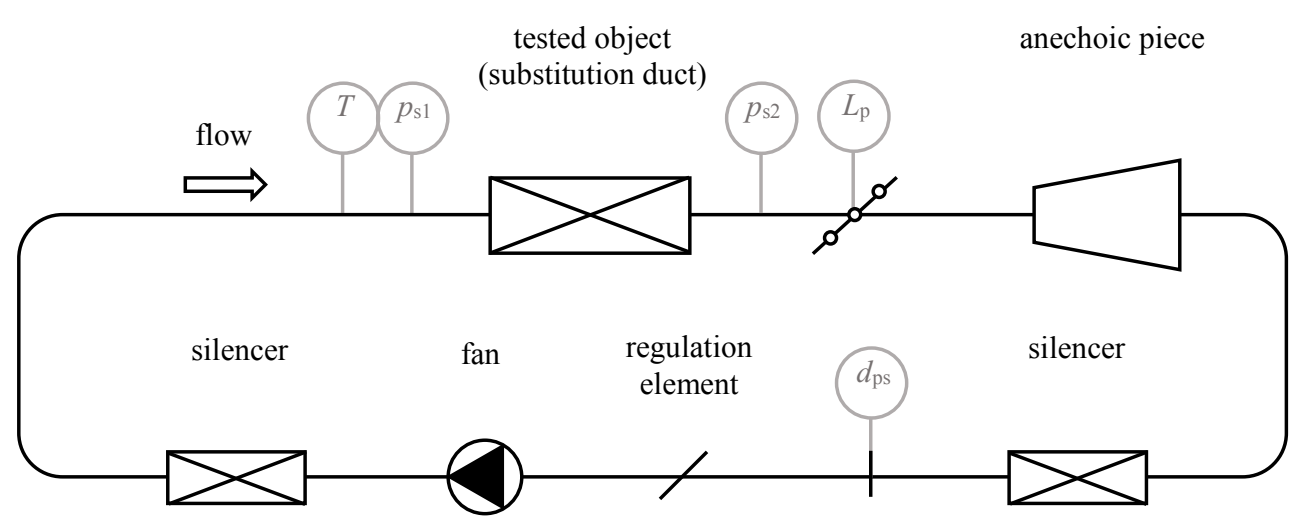

Fig. 1. Scheme of pipeline track without open ends.

anechoic inlet straightener tested object (substitution duct)

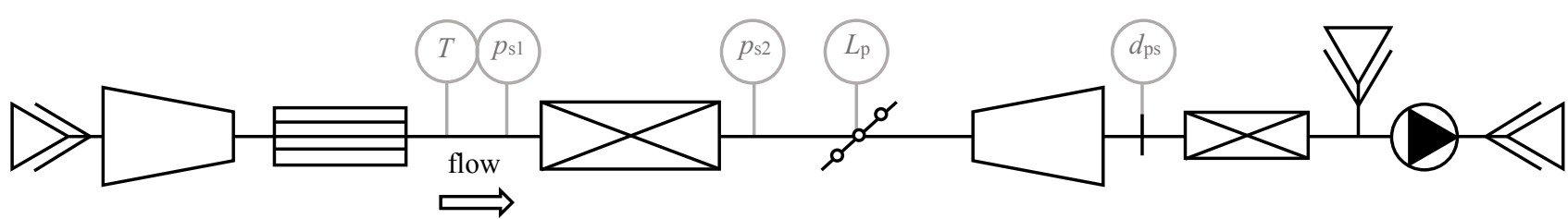

Fig. 2. Scheme of pipeline track with open ends.

flow by a shutter or other throttle element [13]. However, these elements thus incorporated into the air stream can also generate a noise, which in addition will change when its position shifts. And also, if the track is extended, more piping is required.

The second option is the open version of the pipeline track (see Fig. 2). This type of track is relatively simple to build, but it is more space-intensive than the previous one because all the duct elements are placed behind each other and moreover, the track ends must be fitted with anechoic endings. Despite these measures, this type is also more susceptible to the transmission of parasitic noise because of both open ends. On the contrary, the possibility of regulating the flow of air at the inlet or outlet is a big advantage. In the case of configurations, as shown in Fig. 2, it is possible to regulate the flow at 3 locations. The diagram also includes a straightener before the object under test. This element may not be placed there but it may be located in front of the flow measurement.

These tracks configurations also provide the total pressure loss measuring. The suction side airflow measurement has proven to be a better solution, particularly in terms of improved stability of the measured dynamic pressure values $d_{p s}$ [13]. As well as air flow regulation. Looking at Figure 2, it is obvious that the track is built on the suction side. For this type of track, it is necessary to include the regulation element at the inlet. This is due to an increase in the static pressure difference measured before the object under test relative to the air pressure. The position of the control elements at the inlet and the outlet is no longer changed, the flow control is then performed by the T-inlet.

\section{Determining the regenerated noise values}

The basic general relationship is determined by eq. (1). In the case of proper installation of suitable types of anechoic ends, this formula can be modified to

$$
L_{W}=\overline{L_{P}}+K
$$

Measurement of the sound pressure in the pipeline track in a place behind the object under test must be performed in at least three main positions. These microphones must be evenly spaced on the measuring surface at a distance depending on the lowest monitored mid-frequency of the third-octave band. Subsequently, the spatial energy average is calculated for each third-octave band according to the formula

$$
\overline{L_{P}}=10 \log \left[\frac{1}{n} \sum_{i=1}^{n} 10^{0,1 L_{p i}}\right]+C
$$

where

$L_{p i}$ is the time-averaged value of the sound pressure level in decibels at the $\mathrm{i}$-th position;

$C$ is the combined frequency response correction of the microphone sampling tube combination and is given by the formula

$$
C=C_{1}+C_{2}+C_{3,4}
$$

where

$C_{1}$ is the microphone response correction;

$C_{2}$ is the frequency response correction of the microphone shield;

$C_{3,4}$ is the mean flow velocity - modal correction of the microphone shield. 
The individual corrections are determined by the type of cover used against the turbulent flow (sampling tube, nose cone, foam ball). The procedure provides [11].

The $K$ value expresses a constant that is not frequency dependent. This is a value that takes into account the influence of the environment (acoustic impedance) and the cross-sectional area of the pipeline at the place where the sound pressure is measured.

$$
K=L_{p}^{\prime}+10 \log \frac{S}{S_{0}}-10 \log \frac{\rho c}{(\rho c)_{0}}-\overline{L_{P}}
$$

where

$L_{p}^{\prime}$ is the spatial energy average sound pressure corrected for background noise if the background noise value is lower by more than $6 \mathrm{~dB}$ in all of the examined octave bands.

The other variables of this equation (5) refer to the location where the measurement takes place; it is the cross-section area of the pipeline where the microphones are located $S$, the air density $\rho$ and the speed of the sound in the air $c$. The reference value for $S_{0}$ is $1 \mathrm{~m}^{2}$ and for $(\rho c)_{0}$ is $400 \mathrm{~N} \cdot \mathrm{s} / \mathrm{m}^{3}$.

The background noise correction is performed as follows

$$
K_{1 i}=-10 \log \left(1-10^{-0,1 \Delta L_{p i}}\right) .
$$

Where $\Delta L_{p i}=L_{p i}-L_{p i(B)}$ is the difference in the time-averaged sound pressure level during operation and the time-averaged background noise level. The sound background pressure corrected for noise background shall then be calculated according to

$$
L_{p}^{\prime}=L_{p i}-K_{1 i} \text {. }
$$

If background noise correction is not performed, $L_{p}^{\prime}$ is equal to $\overline{L_{P}}$. Consequently, the $L_{W}$ values are given as a chart for each of the third-octave band mid frequencies ranging from $50 \mathrm{~Hz}$ to $10000 \mathrm{~Hz}$ and values less than $0 \mathrm{~dB}$ are not commonly reported.

\section{Conclusion}

Occupant comfort is the goal of all HVAC designers [14]. In terms of meeting hygienic limits to equivalent sound pressure levels in the indoor environment [15], HVAC designers focus primarily on silencer selection as a key part of their HVAC design. However, if a silencer were installed in a duct to reduce noise from the engine room or from the outside and these noises were completely eliminated, a residual noise level would still remain due to the aerodynamic flow [16]. In certain applications and low air velocity, this type of noise is negligible, on the contrary, when the regenerated noise would be comparable to the attenuated sound then this noise would have to be considered. In order to avoid these situations, it is necessary to know the regenerated noise values for certain duct elements.
This article summarizes the information about the regenerated noise measurement in the pipeline track, without a reverberation chamber connection. This method can be used as an alternative to the reverberation chamber method for a large number of duct elements. It should be emphasized that to measure end-of-pipe air-conditioning elements, pipeline measurements are inappropriate. In paper is mentioned the basic philosophy of obtaining the values of regenerated noise by real measurement, as well as the necessary basic equations. In addition, the article contains some recommendations for the track configurations that were obtained on the basis of experimental testing in the Laboratory of Environmental Engineering at FAI TBU in Zlin.

This work was supported by the Ministry of Education, Youth and Sports of the Czech Republic within the National Sustainability Programme project No. LO1303 (MSMT7778/2014) and also by the Internal Grant Agency of Tomas Bata University in Zlin under the project No. IGA/CebiaTech/2018/001.

\section{References}

1. F. Drkal, V. Zmrhal, Větrání, 3 (2013)

2. HVAC Engineering fundamentals, HVAC Systems Design Handbook, 10 (2004)

3. AG 31-010, HVAC Acoustic Fundamentals, 76 (2004)

4. EN ISO 7235, Standard, European Committee for Standardization, Brusel (2009)

5. EN ISO 3741, Standard, European Committee for Standardization, Brusel, (2010)

6. EN ISO 11820, Standard, European Committee for Standardization, Brusel, (1996)

7. M. Möser, G. Müller, Handbook of Engineering Acoustics, (2013)

8. B. Stüber, C. Mühle, K. R. Fritz, Taschenbuch der Technischen Akustik, 2, (1994)

9. EN ISO 14163, Standard, European Committee for Standardization, Brusel, (1998)

10. R. Novy, Hluk a chveni, (2009)

11. EN ISO 5136, Standard, European Committee for Standardization, Brusel, (2009)

12. L. H. Bell, Industrial Noise Control: Fundamentals and Applications, 2, 293 (1993)

13. S. Sehnalek, M. Zalesak, WSEAS Transactions on Systems, 12, 4 (2016)

14. S. K. Wang, Handbook of air conditioning and refrigeration, 2, 926 (2001)

15. EN ISO 15251, Standard, European Committee for Standardization, Brusel, (2011)

16. W. A. Rosenblith, K. N. Stevens, Handbook of acoustic noise control, 2, 183 (1953) 